Equation $\left(416.00^{\prime}\right)$ in reference [2] and equations $(416.00)$ to $(419.00)$ in reference [1] do not contain this term and should be changed.

University of California

Los Alamos Scientific Laboratory

Los Alamos, New Mexico

1. PaUl F. Byrd \& Morris D. Friedman, Handbook of Elliptic Integrals for Engineers and Physicists, Die Grundlehren der Mathematischen Wissenschaften in Einzeldarstellungen, Bd. LXVII, Springer, Berlin, 1954. MR 15, 702.

2. H. A. LAN \& D. F. StEvens, "On the evaluation of certain complex elliptic integrals," Math. Comp., v. 14, 1960, p. 195-199. MR $22 \# 3095$.

\title{
On Davis' Method of Estimating Quadrature Errors
}

\author{
By Y. T. Lo, S. W. Lee, and B. Sun
}

In quadrature method the error is traditionally estimated in terms of the high derivatives of the integrand. The drawbacks of this method are well known. Some ten years ago, Davis [1], Davis and Rabinowitz [2] introduced an interesting new method in estimating the error for analytic functions in terms of their norms. Briefly, for any function $f(z)$ belonging to $L^{2}\left(\varepsilon_{p}\right)$, where $\varepsilon_{p}$ is a region in the complex $z$-plane, bounded by an ellipse with foci at $(-1,0)$ and $(1,0)$, the error $E$ associated with the quadrature

$$
\int_{-1}^{+1} f(x) d x=\sum_{k=0}^{N} a_{k} f\left(\lambda_{k}\right)+E(f)
$$

is bounded by

$$
|E(f)| \leqq \sigma_{R}\|f\|_{\varepsilon_{p}}
$$

In the above relation,

$$
\|f\|_{\varepsilon_{p}}=\left[\iint_{\varepsilon_{p}}|f(z)|^{2} d x d y\right]^{1 / 2}
$$

and $\sigma_{R}$ depends only on the ellipse $\varepsilon_{p}$ and the quadrature rule $R$.

Davis and Rabinowitz [2] have given a short table of $\sigma_{R}$ for a few commonly used quadrature rules and various values of the semi-major axis $a$. To test their results, we have assumed for $f$ a simple trigonometric function whose integral can be easily evaluated and computed. By comparing this with those obtained by various quadratures it turns out that the actual errors are larger than the bound $\sigma_{R}\|f\|_{\varepsilon_{p}}$. Thus it leads us to believe that their tabulated values of $\sigma_{R}$ are in error. In a private communication, Davis agreed with us and encouraged us to recompute their table. Recently we completed this task. The results are tabulated below, where a few more cases and a wider range in semi-major axis $a$ are included. To our disappointment, it is found that these values are much greater than theirs, nearly by a factor of 4 .

Received May 11, 1964. 
This suggests that they might have made an error on the scaling factor. The fact that they do not differ exactly by a factor of 4 for all values seems to be attributed to the truncation error in the infinite series used for evaluating $\sigma_{R}$. The numbers in this table are expressed in floating decimals with the last two digits and $:$ ign referred to the exponent of 10 ; e.g., as $a=1.0001, \sigma_{R}=0.4920 \times 10^{4}$ for the trapezoidal rule.

Antenna Laboratory

University of Illinois

Urbana, Illinois

1. P. Davis, "Errors of numerical approximation for analytic functions," J. Rational Mech. Anal., v. 2, 1953, p. 303-313; Survey of Numerical Analysis, J. ToDd (Ed.), McGraw-Hill,New York, 1962, Chapter 13; "On a problem in the theory of mechanical quadratures," Pacific $J$. Math., v. 5, 1955, p. 669-674. MR 14, 907; MR 24 *B1271; MR 17, 255.

2. P. DAvis \& P. Rabinowitz, "On the estimation of quadrature errors for analytic functions," MTAC, v. 8, 1954, p. 193-203. MR 16, 404. 


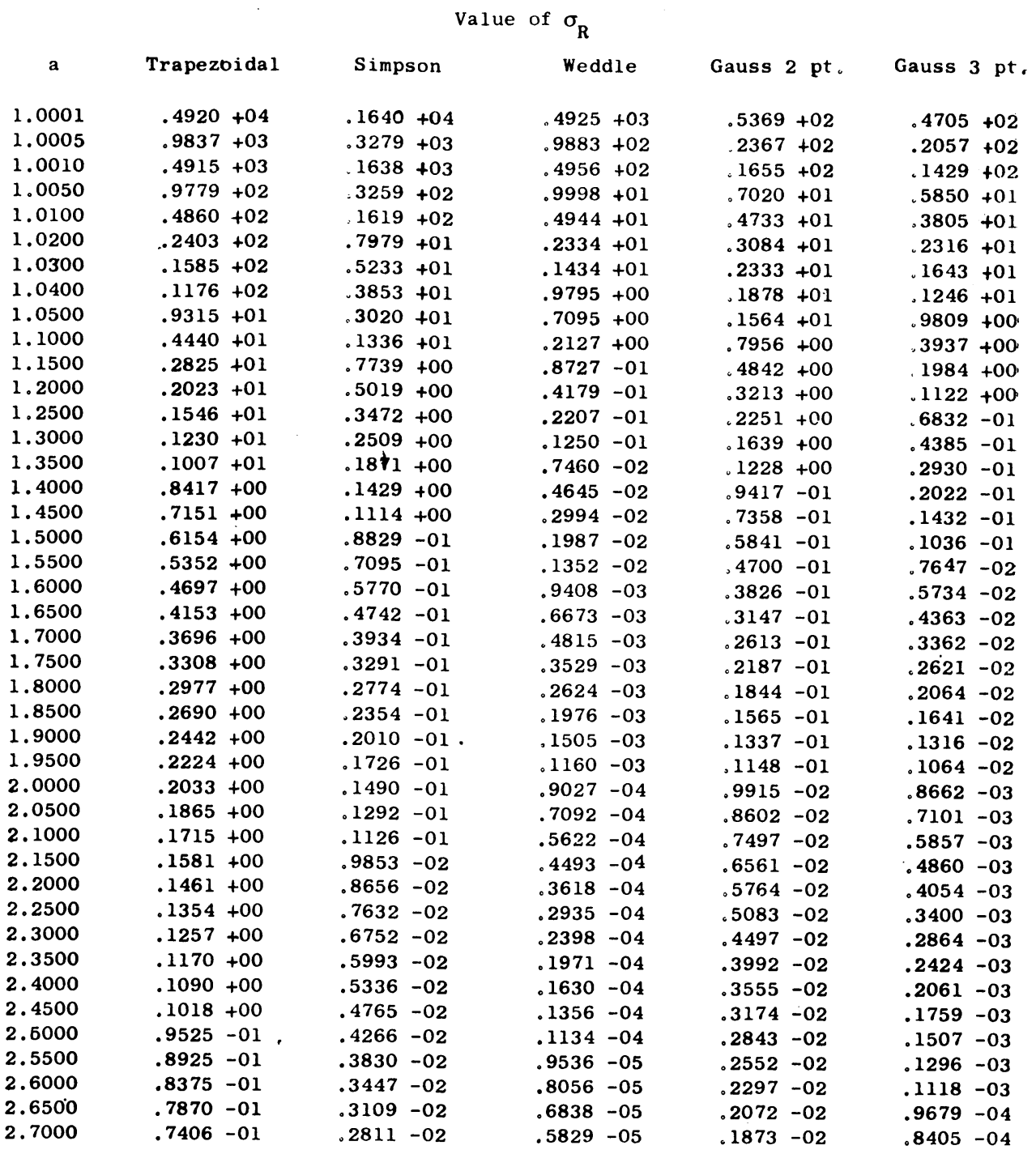


Simpson

$$
\begin{aligned}
& .2547-02 \\
& .2315-02 \\
& .2104-02 \\
& .1917-02 \\
& .1751-02 \\
& .1601-02 \\
& .1467-02 \\
& .1346-02 \\
& .1237-02 \\
& .1138-02 \\
& .1049-02 \\
& .9684-03 \\
& .8950-03 \\
& .8282-03 \\
& .7673-03 \\
& .7118-03 \\
& .6610-03 \\
& .6146-03 \\
& .5720-03 \\
& .5330-03 \\
& .4971-03 \\
& .4641-03 \\
& .4337-03 \\
& .4057-03 \\
& .3798-03 \\
& .3559-03 \\
& .3338-03 \\
& .3134-03 \\
& .2944-03 \\
& .2768-03 \\
& .2604-03 \\
& .2452-03 \\
& .2311-03 \\
& .2179-03 \\
& \text {.2056-03 } \\
& .1942-03 \\
& .1835-03 \\
& .1735-03 \\
& .1641-03 \\
& .1554-03 \\
& .1472-03 \\
& .1395-03 \\
& .1323-03 \\
& .1256-03 \\
& .1192-03 \\
& .1133-03
\end{aligned}
$$

\section{Weddle}

$.4990-05$

$.4288-05$

$.3699-05$

$.3203-05$

$.2783-05$

$.2425-05$

$.2120-05$

$.1859-05$

$.1635-05$

$.1442-05$

$.1275-05$

$.1130-05$

$.1004-05$

$.8937-06$

$.7975-06$

$.7132-06$

$.6391-06$

$.5738-06$

$.5161-06$

$.4651-06$

$.4198-06$

$.3796-06$

$.3438-06$

$.3118-06$

$.2833-06$

$.2577-06$

$.2348-06$

$.2142-06$

$.1957-06$

$.1790-06$

$.1639-06$

$.1503-06$

$.1379-06$

$.1268-06$

$.1166-06$

$.1074-06$

$.9903-07$

$.9139-07$

$.8443-07$

$.7807-07$

$.7226-07$

$.6694-07$

$.6207-07$

$.5760-07$

$.5350-07$

$.4973-07$
Gauss 2 pt. Gauss 3 pt.

$$
\begin{aligned}
& .1697-02 \\
& .1541-02 \\
& .1402-02 \\
& .1278-02 \\
& .1167-02 \\
& .1067-02 \\
& .9776-03 \\
& .8971-03 \\
& .8244-03 \\
& .7588-03 \\
& .6994-03 \\
& .6455-03 \\
& .5966-03 \\
& .5520-03 \\
& .5115-03 \\
& .4745-03 \\
& .4406-03 \\
& .4097-03 \\
& .3813-03 \\
& .3553-03 \\
& .3314-03 \\
& .3094-03 \\
& .2891-03 \\
& .2704-03 \\
& .2532-03 \\
& .2373-03 \\
& .2225-03 \\
& .2089-03 \\
& .1962-03 \\
& .1845-03 \\
& .1736-03 \\
& .1635-03 \\
& .1540-03 \\
& .1453-03 \\
& .1371-03 \\
& .1294-03 \\
& .1223-03 \\
& .1156-03 \\
& .1094-03 \\
& .1036-03 \\
& .9813-04 \\
& .9301-04 \\
& .8821-04 \\
& .8371-04 \\
& .7948-04 \\
& .7551-04
\end{aligned}
$$

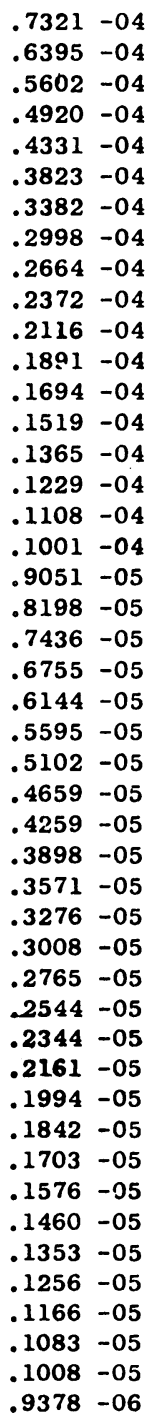




$\begin{array}{llll}1.0001 & .4218+02 & .3304+02 \\ 1.0005 & .1829+02 & .1394+02 \\ 1.0010 & .1261+02 & .9377+01 \\ 1.0050 & .4958+01 & .3135+01 \\ 1.0100 & .3083+01 & .1624+01 \\ 1.0200 & .1725+01 & .6678+00 \\ 1.0300 & .1135+01 & .3408+00 \\ 1.0400 & .8042+00 & .1938+00 \\ 1.0500 & .5954+00 & .1181+00 \\ 1.1000 & .1862+00 & .1713-01 \\ 1.1500 & .7731-01 & .3966-02 \\ 1.2000 & .3719-01 & .1172 & -02 \\ 1.2500 & .1966-01 & .4053 & -03 \\ 1.3000 & .1112-01 & .1569-03 \\ 1.3500 & .6625-02 & .661 \mid & -04 \\ 1.4000 & .4111-02 & .2986 & -04 \\ 1.4500 & .2638-02 & .1426 & -04 \\ 1.5000 & .1742-02 & .7138-05 \\ 1.5500 & .1178-02 & .3720-05 \\ 1.6000 & .8137-03 & .2008 & -05 \\ 1.6500 & .5726-03 & .1118-05 \\ 1.7000 & .4096-03 & .6397-06 \\ 1.7500 & .2974-03 & .3752 & -06 \\ 1.8000 & .2188-03 & .2249-06 \\ 1.8500 & .1629-03 & .1376-06 \\ 1.9000 & .1227-03 & .8576-07 \\ 1.9500 & .9330-04 & .5436-07 \\ 2.0000 & .7164-04 & .3499-07 \\ 2.0500 & .5548-04 & .2285-07 \\ 2.1000 & .4331-04 & .1513-07 \\ 2.1500 & .3407-04 & .1014-07 \\ 2.2000 & .2699-04 & .6877-08 \\ 2.2500 & .2152-04 & .4716-08 \\ 2.3000 & .1727-04 & .3267-08 \\ 2.3500 & .1394-04 & .2285-08 \\ 2.4000 & .1131-04 & .1613-08 \\ 2.4500 & .9224-05 & .1149-08 \\ 2.5000 & .7561-05 & .8249-09 \\ 2.5500 & .6227-05 & .5969-09 \\ 2.6000 & .5151-05 & .4351-09 \\ 2.6500 & .4280-05 & .3195-09 \\ 2.7000 & .3570-05 & .2361-09\end{array}$

$$
\begin{aligned}
& .2773 \quad 02 \\
& .1134+02 \\
& .7395+01 \\
& .1979+01 \\
& .8197+00 \\
& .2399+00 \\
& .9383-01 \\
& .4262-01 \\
& .2130-01 \\
& .1429-02 \\
& .1843-03 \\
& .3345-04 \\
& .7565-05 \\
& .2003-05 \\
& .5978-06 \\
& .1964-06 \\
& .6977-07 \\
& .2648-07 \\
& .1063-07 \\
& .4486-08 \\
& .1976-08 \\
& .9043-09 \\
& .4284-09 \\
& .2093-09 \\
& .1052-09 \\
& .5427-10 \\
& .2866-10 \\
& .1547-10 \\
& .8521-11 \\
& .4782-11 \\
& .2732-11 \\
& .1586-11 \\
& .9351-12 \\
& .5594-12 \\
& \text {.3392 -12 } \\
& .2083-12 \\
& .1295-12 \\
& .8145-13 \\
& .5178-13 \\
& .3327-13 \\
& .2158-13 \\
& .1414-13
\end{aligned}
$$

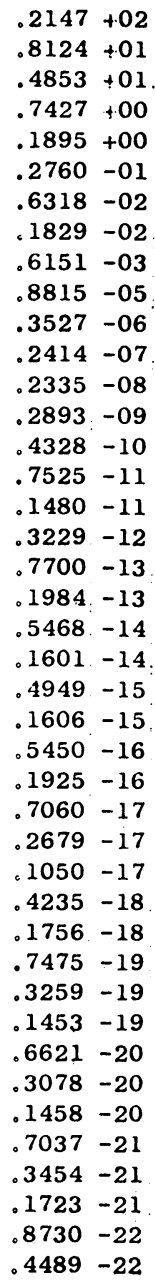




$\begin{array}{lllll}\mathbf{2 . 7 5 0 0} & .2989 & -05 & .1756 & -09 \\ \mathbf{2 . 8 0 0 0} & .2512 & -05 & .1315 & -09 \\ \mathbf{2 . 8 5 0 0} & .2119 & -05 & .9897 & -10 \\ \mathbf{2 . 9 0 0 0} & .1793 & -05 & .7493 & -10 \\ \mathbf{2 . 9 5 0 0} & .1522 & -05 & .5704 & -10 \\ \mathbf{3 . 0 0 0 0} & .1296 & -05 & .4364 & -10 \\ \mathbf{3 . 0 5 0 0} & .1107 & -05 & .3356 & -10 \\ \mathbf{3 . 1 0 0 0} & .9485 & -06 & .2593 & -10 \\ \mathbf{3 . 1 5 0 0} & .8147 & -06 & .2013 & -10 \\ \mathbf{3 . 2 0 0 0} & .7017 & -06 & .1569 & -10 \\ \mathbf{3 . 2 5 0 0} & .6059 & -06 & .1229 & -10 \\ \mathbf{3 . 3 0 0 0} & .5245 & -06 & .9661 & -11 \\ \mathbf{3 . 3 5 0 0} & .4551 & -06 & .7626 & -11 \\ \mathbf{3 . 4 0 0 0} & .3958 & -06 & .6043 & -11 \\ \mathbf{3 . 4 5 0 0} & .3450 & -06 & .4806 & -11 \\ \mathbf{3 . 5 0 0 0} & .3014 & -06 & .3836 & -11 \\ \mathbf{3 . 5 5 0 0} & .2638 & -06 & .3073 & -11 \\ \mathbf{3 . 6 0 0 0} & .2314 & -06 & .2470 & -11 \\ \mathbf{3 . 6 5 0 0} & .2034 & -06 & .1991 & -11 \\ \mathbf{3 . 7 0 0 0} & .1791 & -06 & .1611 & -11 \\ \mathbf{3 . 7 5 0 0} & .1579 & -06 & .1307 & -11 \\ \mathbf{3 . 8 0 0 0} & .1396 & -06 & .1064 & -11 \\ \mathbf{3 . 8 5 0 0} & .1236 & -06 & .8681 & -12 \\ \mathbf{3 . 9 0 0 0} & .1096 & -06 & .7105 & -12 \\ \mathbf{3 . 9 5 0 0} & .9732 & -07 & .5831 & -12 \\ \mathbf{4 . 0 0 0 0} & .8658 & -07 & .4798 & -12 \\ \mathbf{4 . 0 5 0 0} & .7714 & -07 & .3959 & -12 \\ \mathbf{4 . 1 0 0 0} & .6883 & -07 & .3274 & -12 \\ \mathbf{4 . 1 5 0 0} & .6151 & -07 & .2715 & -12 \\ \mathbf{4 . 2 0 0 0} & .5505 & -07 & .2256 & -12 \\ \mathbf{4 . 2 5 0 0} & .4933 & -07 & .1879 & -12 \\ \mathbf{4 . 3 0 0 0} & .4427 & -07 & .1569 & -12 \\ \mathbf{4 . 3 5 0 0} & .3978 & -07 & .1313 & -12 \\ \mathbf{4 . 4 0 0 0} & .3579 & -07 & .1101 & -12 \\ \mathbf{4 . 4 5 0 0} & .3224 & -07 & .9251 & -13 \\ \mathbf{4 . 5 0 0 0} & .2908 & -07 & . .7789 & -13 \\ \mathbf{4 . 5 5 0 0} & .2626 & -07 & .6572 & -13 \\ \mathbf{4 . 6 0 0 0} & .2375 & -07 & 5556 & -13 \\ \mathbf{4 . 6 5 0 0} & .2149 & -07 & .4705 & -13 \\ \mathbf{4 . 7 0 0 0} & .1948 & -07 & .3993 & -13 \\ \mathbf{4 . 7 5 0 0} & .1767 & -07 & .3394 & -13 \\ \mathbf{4 . 8 0 0 0} & .1604 & -07 & .2891 & -13 \\ \mathbf{4 . 8 5 0 0} & .1459 & -07 & .2466 & -13 \\ \mathbf{4 . 9 0 0 0} & .1327 & -07 & .2107 & -13 \\ \mathbf{4 . 9 5 0 0} & .1209 & -07 & .1804 & -13 \\ \mathbf{5 . 0 0 0 0} & .1102 & -07 & .1546 & -13\end{array}$

$$
\begin{aligned}
& .9341-14 \\
& .6226-14 \\
& .4184-14 \\
& .2834-14 \\
& .1934-14 \\
& .1330-14 \\
& .9206-15 \\
& .6416-15 \\
& .4500-15 \\
& .3176-15 \\
& .2255-15 \\
& .1610-15 \\
& .1156-15 \\
& .8349-16 \\
& .6060-16 \\
& .4420-16 \\
& .3240-16 \\
& \text {.2386-16 } \\
& .1765-16 \\
& .1312-16 \\
& .9789-17 \\
& .7336-17 \\
& .5520-17 \\
& .4170-17 \\
& .3162-17 \\
& .2407-17 \\
& .1839-17 \\
& .1409-17 \\
& .1084-17 \\
& .8368-18 \\
& .6479-18 \\
& .5033-18 \\
& .3921-18 \\
& \text {.3065-18 } \\
& .2402-18 \\
& .1888-18 \\
& .1488-18 \\
& .1176-18 \\
& .9323-19 \\
& .7408-19 \\
& .5901-19 \\
& .4713-19 \\
& .3773-19 \\
& .3028-19 \\
& .2435-19 \\
& .1963-19
\end{aligned}
$$

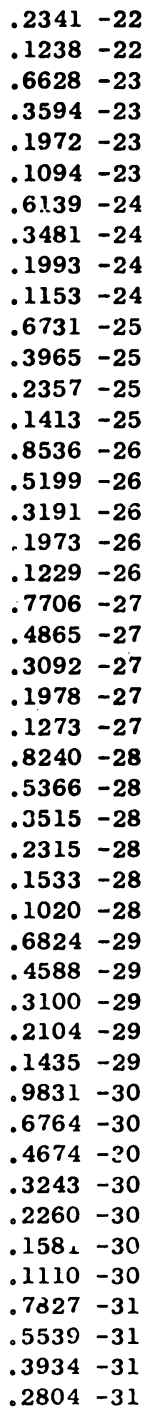

